\title{
CHARACTERIZING HYPERELLIPTIC SURFACES IN TERMS OF CLOSED GEODESICS
}

\author{
Daniel Gallo
}

St. John's University, Staten Island, New York, U.S.A.; gallod@stjohns.edu

\begin{abstract}
We give a characterization of hyperelliptic surfaces in terms of simple, closed geodesics on the surfaces and graphs associated to these geodesics.
\end{abstract}

\section{Introduction and statement of main results}

A closed Riemann surface of genus $g \geq 2$ is hyperelliptic if it has a conformal involution with $2 g+2$ fixed points. Hyperelliptic surfaces were studied classically in the context of function theory on surfaces; a good overview of this approach may be found in [2]. In more recent times, interest has shifted to the geometric structures on the surfaces. In particular, two results, one by Schmutz-Schaller [5], the other by Maskit [4], characterize hyperelliptic surfaces in terms of simple, closed geodesics contained in the surfaces. Later, Costa and Porto [1] obtained the same results by constructing hyperbolic polygons adapted to these geodesics, which also serve to characterize hyperelliptic surfaces. The author is grateful to the referee for pointing out the results in [1].

In this paper the main result is a more general characterization of hyperelliptic surfaces in terms of simple, closed geodesics on the surfaces and graphs associated to these geodesics. As a consequence, we also recover the two results of Schmutz-Schaller and Maskit (Section 4).

In order to state the main theorem precisely, we need the following definition. Let $S$ be a compact surface of genus $g \geq 2$, and let $\mathcal{L}$ be a collection of $2 g-2$ distinct, simple, closed geodesics, $L_{i}, i=1, \ldots, 2 g-2$, contained in $S$. We say $\mathcal{L}$ is evenly spaced if there exists a set of distinct points $X=\left\{x_{1}, \ldots, x_{2 g-1}\right\} \subset S$, called the endpoints of $\mathcal{L}$, such that the following three properties hold:

1) Each geodesic $L_{i} \in \mathcal{L}$ passes through precisely two points of $X$, and the points subdivide $L_{i}$ into two segments of equal length. We call these the endpoints of $L_{i}$.

2) Each point of $X$ lies on some geodesic $L_{i} \in \mathcal{L}$.

Let $\mathcal{T}$ be the abstract graph obtained by taking $2 g-1$ vertices, $\hat{x}_{1}, \ldots, \hat{x}_{2 g-1}$, identifying them with $x_{1}, \ldots, x_{2 g-1}$, respectively, and joining two vertices with an edge $e_{i}$ whenever the two corresponding points in $X$ are the endpoints of the geodesic $L_{i} \in \mathcal{L}$. The graph $\mathcal{T}$ and the collection of geodesics $\mathcal{L}$ will be said to correspond to each other.

3) The graph $\mathcal{T}$ is connected.

Note that, if $\mathcal{L}$ is indeed evenly spaced, then $\mathcal{T}$ is a connected graph with $2 g-1$ vertices and $2 g-2$ edges. It follows that $\mathcal{T}$ is a tree; that is, any two vertices are joined by a unique path.

https://doi.org/10.5186/aasfm.2019.4450

2010 Mathematics Subject Classification: Primary 30F45; Secondary 30F10.

Key words: Fuchsian group, involution, geodesics. 
Theorem 1. Let $\mathcal{T}$ be a tree with $2 g-1$ vertices, $g \geq 2$, and let $S$ be a compact surface of genus $g$. Then $S$ is hyperelliptic if and only if it has an evenly spaced collection of geodesics $\mathcal{L}$ which corresponds to $\mathcal{T}$.

The proof of Theorem 1 will be given in Section 4. The main auxiliary result used in the proof is stated below and proved in Section 3 (see Section 2 for definitions).

Theorem 2. Let $G$ be a Fuchsian group with $\mathbf{U} / G$ a compact surface of genus $g \geq 2$. Suppose $G$ has a proper subgroup $H$ which is precisely embedded and $\mathbf{U} / H$ has an involution $J$ with at least $2 g-1$ fixed points. Then $\mathbf{U} / G$ is hyperelliptic.

\section{Preliminaries}

In this section we establish basic concepts and definitions, and give necessary related results. If a compact surface of genus $g \geq 2$ has a conformal involution with $v>0$ fixed points, then the Riemann-Hurwitz theorem guarantees that there is an integer $0 \leq j \leq \frac{g}{2}$ with

$$
v=2 g+2-4 j .
$$

Equation (1) will be referred to as the Riemann-Hurwitz relation. It is well known that, if the involution is hyperelliptic (that is, with $2 g+2$ fixed points), it is unique. Proofs may be found in [2].

Let $S$ be a compact surface of genus $g \geq 2$. An open subsurface $R \subset S$ will be called a subsurface with geodesic boundary if there exists a non-empty collection $\mathcal{S}$ of simple, closed, disjoint geodesics $l_{1}, \ldots, l_{d}, d>0$, contained in $S$, such that $\bigcup_{1}^{d} l_{i}$ is the boundary in $S$ of $R$. In this case, $R$ is a component of $S-\bigcup_{1}^{d} l_{i}$. Choose disjoint open collars $D_{i} \subset S$ about each $l_{i} \in \mathcal{S}$. The collection of cylinders $R \cap D_{i}$ will be called the ends of $R$. A geodesic $l_{i} \in \mathcal{S}$ is free if $D_{i} \cap R$ has one component. In this case $l_{i}$ is also on the boundary of another component of $S-\bigcup_{1}^{d} l_{i}$. Let $A_{1}, \ldots, A_{k}$ be the complementary components of $R$. Note that $A_{i}, i=1, \ldots, k$, has no boundary components other than those which also lie on the boundary of $R$, so that the boundary of $A_{i}$ consists of free geodesics in $\mathcal{S}$. The corresponding ends of $R$ will be called free ends. If a geodesic $l_{i} \in \mathcal{S}$ is not free, then $D_{i} \cap R$ consists of two components, $l_{i}$ bounds two distinct ends of $R$, and $R \cup l_{i}$ is a surface of genus $g^{\prime}+1$, where $g^{\prime}$ is the genus of $R$. We will call $l_{i}$ a pairing geodesic and each of the the two corresponding ends of $R$ a paired end. If $R$ has $2 p$ distinct paired ends corresponding to $p$ pairing geodesics $l_{1}, \ldots, l_{p}$ in $\mathcal{S}$, then the genus of $\tilde{R}=R \cup l_{1} \cup \cdots \cup l_{p}$ is $g^{\prime}+p$.

Lemma 2.1. Let $S$ be a compact surface of genus $g \geq 2$, and let $R$ be a subsurface with geodesic boundary. Let $g-n$ be the genus of $R, 0<n \leq g$. Suppose $R$ has $r$ ends. Then $r \leq 2 n$.

Proof. Write $r=q+2 p$ where $q$ is the number of free ends and $2 p$ is the number of paired ends. From the discussion above, one has that $\chi(S)=\chi(\tilde{R})+\sum_{1}^{k} \chi\left(A_{i}\right)$ where $\chi$ represents the Euler characteristic. Thus,

$$
2-2 g=\chi(S) \leq \chi(\tilde{R})=2-2(g-n+p)-q .
$$

The inequality follows.

We will work with the upper half-plane model of hyperbolic space given by $\mathbf{U}=$ $\{z \in \mathbf{C}: \operatorname{Im} z>0\}$. Denote the full group of isometries of $\mathbf{U}$ by $P S L(2, \mathbf{R})$, and let $\hat{\mathbf{R}}=\mathbf{R} \cup\{\infty\}$ be the boundary of $\mathbf{U}$. All Fuchsian groups in this paper will be finitely generated, purely hyperbolic, discrete subgroups of $P S L(2, \mathbf{R})$. Given a hyperbolic 
element $\gamma$ in a Fuchsian group, let $l_{\gamma}$ be its axis and $\langle\gamma\rangle$ the cyclic subgroup generated by $\gamma$; it will be assumed throughout that $l_{\gamma}$ is oriented from repelling to attracting fixed point of $\gamma$. We adopt the following convention: a hyperbolic element $\gamma$ in a non-cyclic Fuchsian group $G$ is called a primitive boundary hyperbolic if it generates the stabilizer of $l_{\gamma}$ in $G$, and $l_{\gamma}$ bounds an open half-plane $\mathbf{U}_{\gamma}$, lying to the left of $l_{\gamma}$, which is precisely invariant under $\langle\gamma\rangle$. That is, $g\left(\mathbf{U}_{\gamma}\right) \cap \mathbf{U}_{\gamma}=\emptyset$ for all $g \in G-\langle\gamma\rangle$. In this case, the geodesic $l_{\gamma}$ is called a boundary axis, and the cylinder $\mathbf{U}_{\gamma} /\langle\gamma\rangle$ a boundary cylinder. We will say a Fuchsian group is elementary if it is either trivial or cyclic.

Let $G$ be a Fuchsian group and let $C(G)$ be the smallest hyperbolically closed, convex subset of $\mathbf{U}$ whose Euclidean closure contains the limit set $\Lambda(G)$ of $G$. If $\Lambda(G)=\hat{\mathbf{R}}$, then $C(G)=\mathbf{U}$. If $\Lambda(G) \neq \hat{\mathbf{R}}$ ( $G$ is said to be of the second kind in this case) and $G$ is not elementary, then $C(G)$ is the hyperbolically closed, convex subset of $\mathbf{U}$ bounded by the boundary axes of $G$ (see [4]). In this case, denote the interior of $C(G)$ by $C^{o}(G)$; the convex core is $c(G)=C(G) / G$, and its interior is $c^{o}(G)=C^{o}(G) / G$. If $G$ is a cyclic group, $C(G)$ is a single geodesic, invariant under $G$.

Let $H \subset G$ be a non-elementary subgroup of $G$. Then $H$ is precisely embedded in $G$ if $g\left(C^{o}(H)\right) \cap C^{o}(H)=\emptyset$, for all $g \in G-H$. For $x \in \mathbf{U}$, denote by $[x]_{H}$ and $[x]_{G}$ the respective orbits of $x$. There is a natural covering map, $p_{H}: \mathbf{U} / H \rightarrow \mathbf{U} / G$, given by $p_{H}\left([x]_{H}\right)=[x]_{G}$. The next proposition follows easily from the definitions and the fact that $p_{H}$ is a covering map. We omit the proof.

Proposition 2.2. Let $G$ be a Fuchsian group with $\mathbf{U} / G$ compact, and let $H$ be a non-elementary, proper subgroup which is precisely embedded in $G$. Then $p_{H}$ maps $c^{o}(H)$ injectively into $\mathbf{U} / G$. Moreover, $p_{H}$ maps the boundary of $c^{o}(H)$ onto a collection of disjoint, simple, closed geodesics which are the boundary of $p_{H}\left(c^{o}(H)\right)$. Consequently, $p_{H}\left(c^{o}(H)\right)$ is a subsurface with geodesic boundary in $\mathbf{U} / G$.

If $H$ is cyclic, we say that it is precisely embedded in the Fuchsian group $G$ if $H$ is the stabilizer in $G$ of $C(H)$ and $C(H)$ projects to a simple, closed geodesic in $\mathbf{U} / G$. The trivial group is precisely embedded in every group. For $j \in P S L(2, \mathbf{R})$ an elliptic element of order two, a subgroup $H \subset P S L(2, \mathbf{R})$ is $j$-invariant if $j H j=H$. The following is proved in [4]:

Proposition 2.3. Let $G$ be a Fuchsian group and let $j \in P S L(2, \mathbf{R})$ be an elliptic element of order two. Let $H_{1}, H_{2}$ be nontrivial, $j$-invariant, precisely embedded subgroups of $G$. Then there is a $j$-invariant, precisely embedded subgroup $H \subset G$ with $H_{1} \cup H_{2} \subset H$.

\section{Open surfaces with involutions}

The main result of this section is Theorem 2. Before proving it, we establish basic properties of open surfaces with involutions, and recall the construction of amalgamated free products and HNN extensions of Fuchsian groups (details may be found in [3]). Note first that one sees easily that, if $G$ is a non-elementary Fuchsian group of the second kind and $J$ is an involution of $\mathbf{U} / G$, then the fixed points of $J$ are contained in $c^{o}(G)$.

Lemma 3.1. Let $G$ be a non-elementary Fuchsian group of the second kind with $\gamma \in G$ a primitive boundary hyperbolic. Suppose $\mathbf{U} / G$ has an involution $J$ which fixes the boundary cylinder $\mathbf{U}_{\gamma} /\langle\gamma\rangle$. Then $J$ has a lift $\zeta \in P S L(2, \mathbf{R})$ with $\zeta^{2}=\gamma$. 
Proof. Let $h \in P S L(2, \mathbf{R})$ be an arbitrary lift of $J$. Since $J$ leaves $\mathbf{U}_{\gamma} /\langle\gamma\rangle$ invariant, it follows that there exists $T \in G$ with $T h\left(l_{\gamma}\right)=l_{\gamma}$. Hence, $T h$ leaves $l_{\gamma}$ invariant. Since $T h$ is also a lift of $J$, we have that $(T h)^{2}$ lies in $G$, and it must be a power of $\gamma$. We assume $(T h)^{2}=\gamma^{s}$, where $s>0$ (the proof is similar if $s<0$ ). Necessarily, $s$ is an odd integer, otherwise $T h$ lies in $G$, contradicting that $T h$ is the lift of an involution. Since $T h=\gamma^{\frac{s-1}{2}} \gamma^{\frac{1}{2}}$ and $\gamma^{\frac{s-1}{2}} \in G$, it follows that $T h$ and $\zeta=\gamma^{\frac{1}{2}}$ induce the same involution.

An open surface is of type $(g, m)$ if, topologically, it can be obtained from a compact surface of genus $g$ by removing $m$ disjoint closed discs. A Fuchsian group $G$ is of type $(g, m)$ whenever $\mathbf{U} / G$ is a surface of type $(g, m)$. Let $H$ be a Fuchsian group of type $(1,1)$. It is well known that $\mathbf{U} / H$ has a unique involution $J_{1}$ which fixes three points and the one boundary cylinder of $\mathbf{U} / H$ (see [4] for details). Let $\beta \in H$ be a primitive boundary hyperbolic.

The amalgamated free product. Suppose $G$ is a non-elementary Fuchsian group of type $(g, m)$ with $\beta^{-1} \in G$ a primitive boundary hyperbolic. The group $H *_{\beta} G$ generated by $H$ and $G$ is called an amalgamated free product; it is discrete and has type $(g+1, m-1)$. Each primitive boundary hyperbolic in $G$ that is not equivalent to $\beta^{-1}$ remains a primitive boundary hyperbolic in $H *_{\beta} G$. Topologically, the surface $\mathbf{U} /\left(H *_{\beta} G\right)$ is obtained by attaching $(\mathbf{U} / G)-\left(\mathbf{U}_{\beta^{-1}} /\langle\beta\rangle\right)$ to $(\mathbf{U} / H)-\left(\mathbf{U}_{\beta} /\langle\beta\rangle\right)$ at the common closed geodesic $l_{\beta} /\langle\beta\rangle$.

The HNN extension. Let $G$ be a non-elementary Fuchsian group of type $(g, m)$. Suppose there exist distinct primitive boundary hyperbolics $\eta, \varphi \in G$ and $\gamma \in P S L(2, \mathbf{R})$ with $\gamma^{-1} \eta \gamma=\varphi^{-1}$. Then the group $G *_{\gamma}$ generated by $G$ and $\gamma$ is called an HNN extension of $G$; it is discrete and has type $(g+1, m-2)$. Each primitive boundary hyperbolic in $G$ that is not equivalent to either $\eta$ or $\varphi$ remains a primitive boundary hyperbolic in $G *_{\gamma}$. Topologically, the surface $\mathbf{U} / G *_{\gamma}$ is obtained by joining the two closed, oriented geodesics $l_{\eta} /\langle\eta\rangle, l_{\varphi^{-1}} /\langle\varphi\rangle$ in $(\mathbf{U} / G)-\left(\mathbf{U}_{\eta} /\langle\eta\rangle \cup \mathbf{U}_{\varphi} /\langle\varphi\rangle\right)$.

Lemmas 3.2 and 3.3, which follow, are technical statements necessary for the proof of Proposition 3.4. We have chosen to highlight them rather than include their texts in the proof.

Lemma 3.2. (Attaching a one-holed torus) Let $H$ be a Fuchsian group of type $(1,1)$, and $\beta \in H$ a primitive boundary hyperbolic. Let $J_{1}$ be the natural involution on $\mathbf{U} / H$. Suppose $G$ is a non-elementary Fuchsian group of the second kind such that $\mathbf{U} / G$ has an involution $J_{2}$ with $v \geq 0$ fixed points. Further, suppose $G$ is of type $(g, k+2 m)$ where $k$ is the number of boundary cylinders fixed by $J_{2}$ and $2 m$ is the number of boundary cylinders interchanged by $J_{2}$ (in pairs). Let $\beta^{-1}$ be a primitive boundary hyperbolic in $G$ corresponding to a fixed boundary cylinder. Then $H *_{\beta} G$ has type $(g+1, k-1+2 m)$, and $\mathbf{U} /\left(H *_{\beta} G\right)$ has an involution with $v+3$ fixed points, $k-1$ fixed boundary cylinders, and $2 m$ boundary cylinders which are interchanged.

Proof. By Lemma 3.1, $J_{1}$ and $J_{2}$ lift to $\zeta \in P S L(2, \mathbf{R})$ where $\zeta^{2}=\beta$. Since $\zeta$ normalizes both $H$ and $G$, it also normalizes $H *_{\beta} G$ and projects to an involution of $\mathbf{U} /\left(H *_{\beta} G\right)$ having $v+3$ fixed points; the remaining assertions are clear.

Lemma 3.3. (Closing a handle) Let $G$ be a non-elementary Fuchsian group of the second kind, of type $(g, 2 m)$, where $\mathbf{U} / G$ has an involution $J$ which interchanges the $2 m$ boundary cylinders and has $v>0$ fixed points. Let $\eta, \varphi \in G$ be distinct, primitive boundary hyperbolics which correspond to boundary cylinders interchanged 
by $J$. Let $\gamma \in P S L(2, \mathbf{R})$ with $\gamma^{-1} \eta \gamma=\varphi^{-1}$. Then $G *_{\gamma}$ is of type $(g+1,2 m-2)$, and $\mathbf{U} / G *_{\gamma}$ has an involution which fixes $v+2$ points and interchanges the $2 m-2$ boundary cylinders.

Proof. Let $j$ be an elliptic lift of $J$ with fixed point $a \in C^{o}(G)$. We assume without loss of generality that $j\left(l_{\eta}\right)=l_{\varphi}$ and $j \eta j=\varphi$. Let $l$ be the smallest closed geodesic segment perpendicular to $l_{\eta}$ and $l_{\varphi}$ which joins the two axes. Then the fixed point $a$ of $j$ is the midpoint of $l$. One sees easily that $l_{\gamma}$ must pass through $a$ and cross both $l_{\eta}$ and $l_{\varphi}$. Thus, $j \gamma j=\gamma^{-1}$ and $j\left(G *_{\gamma}\right) j=G *_{\gamma}$, so that $j$ projects to an involution of $\mathbf{U} / G *_{\gamma}$. Let $a_{1}, a_{2}$ be the intersection points of $l_{\gamma}$ with $l_{\eta}, l_{\varphi}$, respectively. Clearly, since $j\left(a_{1}\right)=a_{2}$ and $\gamma\left(a_{1}\right)=a_{2}, a_{1}$ projects to a fixed point. Letting $\varphi^{\frac{1}{2}}\left(a_{2}\right)=b$, one verifies easily that that $\varphi \gamma j(b)=b$, so that $b$ projects to another fixed point. The remaining assertions are clear.

Proposition 3.4. Let $G$ be a Fuchsian group with $\mathrm{U} / G$ a compact surface of genus $g \geq 2$. Suppose $G$ has a proper subgroup $H$ which is precisely embedded and $R=\mathbf{U} / H$ has a involution $J$ with at least $2 g-1$ fixed points. Then, either

1) $R$ is of type $(g-1,1)$, and $J$ has $2 g-1$ fixed points; or

$2) R$ is of type $(g-1,2)$, and $J$ has either $2 g-1$ or $2 g$ fixed points. Moreover, $J$ interchanges the boundary cylinders of $R$.

Proof. One sees easily, since $J$ has $2 g-1 \geq 3$ fixed points, that $H$ is not elementary. Necessarily, then, $\mathbf{U} / H$ is of type $(g-n, k+2 m), 0<n \leq g, 0 \leq k$, $0 \leq m, 0<k+2 m$, with $k$ boundary cylinders fixed by $J$ and $2 m$ boundary cylinders interchanged by $J$. Since $\mathbf{U} / H$ has the same type as $c^{o}(H)$, it follows from Lemmas 2.1 and 2.2 that

$$
0<k+2 m \leq 2 n .
$$

Let $N \geq 2 g-1$ be the number of fixed points of $J$. Starting with $\mathbf{U} / H$, we will use amalgamated free products (attaching $k$ one holed tori) and HNN extensions (closing $m$ handles) to construct a compact surface $S^{\prime}$ of genus $g-n+k+m$ having an involution with $N+3 k+2 m$ fixed points.

Let $\beta_{i}, i=1, \ldots, k$, be primitive boundary hyperbolics in $H$ corresponding to the boundary cylinders fixed by $J$. We choose $k$ one-holed torus groups $H_{i}$ with primitive boundary hyperbolics $\beta_{i}^{-1}$, and use Lemma 3.2 and an inductive argument to

a) construct the group $B=H *_{\beta_{1}} H_{1} *_{\beta_{2}} \cdots *_{\beta_{k}} H_{k}$, and

b) show that $\mathbf{U} / B$ is a surface of type $(g-n+k, 2 m)$ having an involution with $N+3 k$ fixed points which interchanges the $2 m$ boundary cylinders.

Let $\eta_{1}, \ldots, \eta_{m}, \varphi_{1}, \ldots, \varphi_{m}$ be primitive boundary hyperbolics in $B$, where $\mathbf{U}_{\eta_{i}} /\left\langle\eta_{i}\right\rangle$ and $\mathbf{U}_{\varphi_{i}} /\left\langle\varphi_{i}\right\rangle, i=1, \ldots, m$, are interchanged. Choose hyperbolic elements $\gamma_{i} \in$ $P S L(2, \mathbf{R})$ with $\gamma_{i}^{-1} \eta_{i} \gamma_{i}=\varphi_{i}^{-1}$. We use Lemma 3.3 and an inductive argument to

a) construct the group $C=B *_{\gamma_{1}} \cdots *_{\gamma_{m}}$, and

b) show that $S^{\prime}=\mathbf{U} / C$ is a compact surface of genus $g-n+k+m$ having an involution with $N+3 k+2 m$ fixed points.

By the Riemann-Hurwitz relation, the involution on $S^{\prime}$ has at most $2(g-n+$ $k+m)+2$ fixed points. Thus,

$$
2(g-n+k+m)+2 \geq N+3 k+2 m .
$$

If $N=2 g-1$, then the only solutions to the inequality above are either 
1) $n=1, k=1$ (and from (3), $m=0$ ), so that $\mathbf{U} / H$ is of type $(g-1,1)$; or

2) $n=1, k=0$ (and from (3), $m=1$ ), so that $\mathbf{U} / H$ is of type $(g-1,2)$.

If $N=2 g$, then the only solution is

3) $n=1, k=0$ (and from (3), $m=1$ ), so that $\mathbf{U} / H$ is of type $(g-1,2)$. If $N>2 g$, then (4) has no solutions.

Proof of Theorem 2. In view of Proposition 3.4, we need only consider two cases.

Case 1) $R$ is of type $(g-1,1)$, and $J$ has $2 g-1$ fixed points. Here, $p_{H}\left(c^{o}(H)\right)$ has one complementary component $A \subset \mathbf{U} / G$, necessarily a surface of type $(1,1)$ sharing a boundary geodesic $l$ with $p_{H}\left(c^{o}(H)\right)$. Let $\beta \in H$ be a primitive boundary hyperbolic. Choose $B$ a component of $p_{1}^{-1}(A)$ where the closure $\bar{B}$ contains $l_{\beta}$ ( $p_{1}: \mathbf{U} \rightarrow \mathbf{U} / G$ is the natural covering). Let $H_{1} \subset G$ be the stabilizer of $B$; it follows from [4] (Props. 4.1, 4.2) that $H_{1}$ is precisely embedded, hence finitely generated. One sees easily that $H_{1}$ is not elementary and that $C\left(H_{1}\right)=\bar{B}$.

Now $B=C^{o}\left(H_{1}\right)$, and $\mathbf{U} / H_{1}$ has the same type as $B / H_{1}=A$; consequently, $H_{1}$ is a group of type $(1,1)$. Trivially, since $l_{\beta}$ is a boundary component for both $C(H)$ and $C\left(H_{1}\right), \beta^{-1}$ is a primitive boundary hyperbolic in $H_{1}$. Thus, $H *_{\beta} H_{1}$ is a group of type $(g, 0)$ and $H *_{\beta} H_{1}=G$. By Lemma $3.2, \mathbf{U} / G$ has an involution with $2 g+2$ fixed points.

Case 2) $R$ is of type $(g-1,2)$, and $J$ has either $2 g-1$ or $2 g$ fixed points. Let $\eta, \varphi \in H$ be primitive boundary hyperbolics which correspond to the two boundary cylinders of $R$ which are interchanged by $J$. Here, $p_{H}\left(c^{o}(H)\right)$ has no complementary components, and necessarily, $p_{H}\left(l_{\eta} /\langle\eta\rangle\right)=p_{H}\left(l_{\varphi^{-1}} /\langle\varphi\rangle\right)$. It follows that there exists $T \in G$ with $T^{-1} \eta T=\varphi^{-1}$, so that $G=H *_{T}$. By Lemma $3.3, \mathbf{U} / G$ has an involution with either $2 g+1$ or $2 g+2$ fixed points. If it has $2 g+1$ fixed points, then by the Riemann-Hurwitz relation, it must have $2 g+2$ fixed points.

\section{The characterization theorem}

We prove Theorem 1 in the next two propositions.

Proposition 4.1. Let $\mathcal{T}$ be a tree with $2 g-1$ vertices, $g \geq 2$, and let $S$ be a compact surface of genus $g$ with an evenly spaced collection of geodesics $\mathcal{L}$ which corresponds to $\mathcal{T}$. Then $S$ is hyperelliptic.

Proof. Let $G$ be a Fuchsian group with $\mathbf{U} / G=S$, and fix $\hat{x}_{1}$ an arbitrary vertex in $\mathcal{T}$. The idea of the proof is the following: Let $P_{1}, \ldots, P_{s}$ be the collection of maximal paths in $\mathcal{T}$ based at $\hat{x}_{1}$. We will construct for each $P_{k}, k=1, \ldots, s$, a subgroup $M_{k} \subset G$ which is precisely embedded and $j$-invariant for a fixed elliptic element $j$ of order two. Applying Proposition 2.3, we then obtain a precisely embedded, $j$ invariant subgroup $H$ with $M_{1} \cup \cdots \cup M_{s} \subset H$. After showing that the natural involution on $\mathbf{U} / H$ has at least $2 g-1$ fixed points, we invoke Theorem 2 and prove that $\mathbf{U} / G$ is hyperelliptic.

Let $\hat{x}_{1}, \ldots, \hat{x}_{n}, n \geq 2$, be the ordered vertices of a maximal path $P_{k}$ in $\mathcal{T}$. Let $l_{i}^{\prime}$ be the edges of the path, where $l_{i}^{\prime}$ joins $\hat{x}_{i}$ to $\hat{x}_{i+1}, i=1, \ldots, n-1$. Let $x_{1}, \ldots, x_{n} \in S$ be the corresponding endpoints of $\mathcal{L}$ and $L_{i} \subset S, i=1, \ldots, n-1$, the simple, closed geodesics in $\mathcal{L}$ corresponding to $l_{i}^{\prime}$.

Let $z_{1}$ be an arbitrary lift of $x_{1}$ to $\mathbf{U}$, and choose a hyperbolic element $T_{1} \in G$ which generates the cyclic subgroup corresponding to $L_{1}$ and has axis $l_{1}$ passing through $z_{1}$. Let $z_{i}, i=2, \ldots, n$, be the midpoint of the segment between $z_{i-1}$ and 
$T_{i-1}\left(z_{i-1}\right)$ where, for $n>2$ and $i=2, \ldots, n-1, T_{i} \in G$ is a hyperbolic element which generates the cyclic subgroup corresponding to $L_{i}$ and has axis $l_{i}$ passing through $z_{i}$. Note that the $z_{i}, i=1, \ldots, n$, are lifts of the elements $x_{i}$.

Let $j_{1}$ be the elliptic transformation of order two fixing $z_{1}$. Then

$$
j_{i}=T_{i-1} j_{i-1}=T_{i-1} \cdots T_{1} j_{1},
$$

is the elliptic transformation of order two fixing the point $z_{i}, i=2, \ldots, n$. Note that, since $z_{i}$ lies on $l_{i}$, for $i=1, \ldots, n-1$, we have that

$$
j_{i} T_{i} j_{i}=T_{i}^{-1} \text {. }
$$

Let $G_{i}$ be the group generated by $T_{1}, \ldots, T_{i}$. We now show that there exists a precisely embedded, $j_{1}$-invariant subgroup $K_{n-1}$ of $G$ with $G_{n-1} \subset K_{n-1}$. The proof is by induction on $i$.

Clearly, since $T_{1}$ corresponds to a simple, closed geodesic in $\mathbf{U} / G,\left\langle T_{1}\right\rangle=K_{1}$ is precisely embedded in $G$. It is $j_{1}$-invariant by (6). Let $K_{i}$ be a precisely embedded, $j_{1}$-invariant subgroup, with $G_{i} \subset K_{i} \subset G, i<n-1$. From (5), since $T_{1}, \ldots, T_{i} \in K_{i}$, we have that $j_{i+1}$ and $j_{1}$ project to the same involution of $\mathbf{U} / K_{i}$. Hence, $K_{i}$ is $j_{i+1}$-invariant. Since $T_{i+1}$ corresponds to a simple, closed geodesic in $\mathbf{U} / G,\left\langle T_{i+1}\right\rangle$ is precisely embedded. Moreover, from (6), we also have that $\left\langle T_{i+1}\right\rangle$ is $j_{i+1}$-invariant. Applying Proposition 2.3 to $K_{i}$ and $T_{i+1}$, there exists a precisely embedded group $K_{i+1}$ which is $j_{i+1}$-invariant, with $K_{i} \cup\left\langle T_{i+1}\right\rangle \subset K_{i+1}$. From (5), since $G_{i+1} \subset K_{i+1}$, it follows that $K_{i+1}$ is $j_{1}$-invariant.

Let $M_{k}=K_{n-1}$. We repeat the process for each maximal path based at $\hat{x}_{1}$ in $\mathcal{T}$ (lifting $x_{1}$ to $z_{1}$ each time). Thus, for each such path $P_{k}, k=1, \ldots, s$, there is a precisely embedded, $j_{1}$-invariant subgroup $M_{k}$. If $s>1$, we apply Proposition 2.3 $s-1$ times and obtain a precisely embedded, $j_{1}$-invariant subgroup $H$ with $M_{1} \cup$ $\cdots \cup M_{s} \subset H$. If $s=1, H=M_{1}$. Let $J$ be the induced involution of $U / H$.

Each endpoint $x_{i}$ of $\mathcal{L}$ corresponds to some vertex $\hat{x}_{i}$ in at least one of the maximal paths, say $P_{k}$, of $\mathcal{T}$. In the construction of $M_{k}$, we chose a lift $z_{i} \in \mathbf{U}$ of $x_{i}$ which projects to a fixed point of $J$ in $\mathbf{U} / H$. Since these lifts of endpoints of $\mathcal{L}$ project to $2 g-1$ distinct points of $\mathbf{U} / G$, they project to at least $2 g-1$ distinct points of $\mathbf{U} / H$. It follows that $J$ has at least $2 g-1$ fixed points.

If $H$ is a proper subgroup of $G$, then by Theorem $2, \mathbf{U} / G$ is hyperelliptic. On the other hand, if $H=G$, then $J$ is an involuton of $\mathbf{U} / G$ with at least $2 g-1$ fixed points. By the Riemann-Hurwitz relation, the involution must have $2 g+2$ fixed points, and $\mathbf{U} / G$ is hyperelliptic.

Proposition 4.2. Let $\mathcal{T}$ be a tree with $2 g-1$ vertices, $g \geq 2$, and let $S$ be a hyperelliptic surface of genus $g$. Then $S$ has an evenly spaced collection of geodesics $\mathcal{L}$ which corresponds to $\mathcal{T}$.

Proof. Let $S=\mathrm{U} / G$ with $G$ a Fuchsian group, and let $J$ be the hyperelliptic involution. Then $S /\langle J\rangle$ is a sphere with $2 g+2$ distinguished points which are the projections, $w_{1}, \ldots, w_{2 g+2}$, of the fixed points of $J, x_{1}, \ldots, x_{2 g+2}$, respectively. The ordering of these points is arbitrary. It is well known that any tree is planar, that is, when viewed as a topological space, it can be embedded in the sphere. We choose a realization of $\mathcal{T}$ with $w_{1}, \ldots, w_{2 g-1}$ as vertices; the edges are given by simple curves $s_{i} \subset S / J, i=1, \ldots, 2 g-2$, with no two curves intersecting, except possibly at their endpoints.

Since $J$ is a twofold cover, branched to order two over each of the points $w_{1}, \ldots$, $w_{2 g-1}$, each curve $s_{i}$ lifts to a simple, closed curve $s_{i}^{\prime}$ in $S$ passing through two 
fixed points, which we denote $y_{i}, y_{i+1} \in\left\{x_{1}, \ldots, x_{2 g-1}\right\}$. Let $z_{i} \in \mathbf{U}$ be a lift of $y_{i}$ and let $S_{i} \subset \mathrm{U}$ be a component of the preimage of $s_{i}^{\prime}$ (under the natural covering $\left.p_{1}: \mathbf{U} \rightarrow \mathbf{U} / G\right)$ passing through $z_{i}$. Since $s_{i}^{\prime}$ is a simple, closed curve, $S_{i}$ is invariant under a cyclic subgroup of $G$ generated by some element $T_{i} \in G$. Choose $z_{i+1} \in S_{i}$, a lift of $y_{i+1}$, and let $j_{i}$ (resp. $j_{i+1}$ ) be the elliptic transformation of order two, necessarily a lift of $J$, with fixed point $z_{i}$ (resp. $z_{i+1}$ ). Since $S_{i}$ is invariant under both $j_{i}$ and $j_{i+1}$, it follows that it is invariant under their product $R_{i}=j_{i+1} j_{i} \in G$, and $R_{i}=T_{i}^{n}$ with $n \neq 0$. Hence, $s_{i}^{\prime}$ is homotopic to the the simple, closed geodesic $L_{i}$ given by $L_{i}=l_{R_{i}} /\left\langle T_{i}\right\rangle$. Note that, since $z_{i+1}$ lies halfway between $z_{i}$ and $T_{i}^{n}\left(z_{i}\right)$, and $z_{i}, z_{i+1}$ are inequivalent in $G, n$ must be an odd number. Without loss of generality, we assume $n$ is positive.

Letting $j_{i}^{\prime}=T_{i} j_{i}$, one has that $z_{i}^{\prime}$, the fixed point of $j_{i}^{\prime}$, lies halfway between $z_{i}$ and

$T_{i}\left(z_{i}\right)$. Hence, $z_{i+1}=T_{i}^{\frac{n-1}{2}}\left(z_{i}^{\prime}\right)$ and $z_{i+1}$ is equivalent to $z_{i}^{\prime}$. It follows that $z_{i}^{\prime}$ is a lift of $y_{i+1}$. Thus, $y_{i}, y_{i+1}$ divide $L_{i}$ into two segments of equal length. Noting that the $L_{i}$ have the same points of intersection as the $s_{i}^{\prime}$, we have that $L_{i}, i=1, \ldots, 2 g-2$, along with the points $x_{1}, \ldots, x_{2 g-1}$, form an evenly spaced collection of geodesics corresponding to $\mathcal{T}$.

Let $S$ be a compact surface of genus $g \geq 2$, and let $L_{1}, \ldots, L_{2 g-2} \subset S$ be simple, closed geodesics which intersect at a single point $p \in S$ and are otherwise disjoint. Let $p_{i}$ be the midpoint of the geodesic $L_{i}$, viewed as a geodesic from $p$ to $p$. Then the geodesics $L_{i}$ with the points $p, p_{i}, i=1, \ldots, 2 g-2$, form a collection of evenly spaced geodesics whose corresponding graph is a tree. The next result now follows from Theorem 1.

Theorem 3. (Schmutz-Schaller [5]) Let $S$ be a compact surface of genus $g \geq 2$. Then $S$ is hyperelliptic if and only if it has at least $2 g-2$ simple, closed geodesics which intersect at one point and are otherwise disjoint.

A necklace with $2 g-2$ successive evenly spaced links on a compact surface of genus $g \geq 2$ is an evenly spaced set of $2 g-2$ geodesics $\mathcal{L}$ whose corresponding graph $\mathcal{T}$ is a path. The following is also obtained from Theorem 1.

Theorem 4. (Maskit [4]) Let $S$ be a compact surface of genus $g \geq 2$. Then $S$ is hyperelliptic if and only if it has a necklace with $2 g-2$ successive evenly spaced links.

\section{References}

[1] Costa, A., and A. Porto: On two recent geometrical characterizations of hyperellipticity. Rev. Mat. Complut. 17:1, 2004, 59-65.

[2] Farkas, H. M., and I. Kra: Riemann surfaces. - Springer-Verlag, New York, 1980.

[3] Maskit, B.: Kleinian groups. - Springer-Verlag, Berlin, 1988.

[4] Maskit, B.: A new characterization of hyperellipticity. - Michigan Math. J. 47:1, 2000, 3-14.

[5] Schmutz-Schaller, P.: Geometric characterization of hyperelliptic Riemann surfaces. - Ann. Acad. Sci. Fenn. Math. 25, 2000, 85-90. 